\title{
The Focused Ion Beam Hard Mask Mechanism
}

\author{
W.R. McKenzie* and P.R. Munroe* \\ * Electron Microscope Unit, University of New South Wales, Sydney NSW 2052, Australia
}

The Focused Ion Beam Hard Mask (FIBHM) method has recently been established as new form of direct-write ion beam lithography suited for high resolution patterning of diamond [1]. Diamond's combination of extreme material and functional properties make it potentially useful for a large array of new applications [2-3]. Some of these properties include the highest Young's modulus, highest thermal conductivity and number density of atoms. It is also biocompatible and can to emit photons at room temperature. The FIBHM has provided a means to realize some such applications including: patterning high resolution dies for nanoimprint lithography [1, 4] and for patterning nano-scaled waveguides suited for integrated optical circuits and potentially q-bits for quantum computing [5-6].

The FIBHM method is outlined in Fig. 1(A) to produce structures such as those shown in the TEM cross-section shown in Fig. 1(B). Step 1 involves an exposure to a naked diamond surface using a focused ion beam (FIB) (e.g. $\left.>10^{16} \mathrm{Ga}^{+} / \mathrm{cm}^{2} 30 \mathrm{kV}\right)$ over the area to be masked. Step 2 involves a plasma etch containing either oxygen or argon containing atmosphere to etch the unmasked diamond. The introduction of reactive gas species, such as $\mathrm{CHF}_{3}$ (with oxygen) has been found to introduce a lateral component to the etch allowing the fabrication of sloped sidewalls leading to the trapezoidal structures shown in Fig. 1(B).

The masking mechanism differs from the existing understanding of masking in reactive ion etch environments given its effectiveness against an inert Ar plasma etch [5]. For the FIBHM the masking effect is believed to be a result of an additional layer grown from impurities in the plasma seeded by the $\mathrm{Ga}^{+}$implanted surface. Fig. 2(A) shows a bright field STEM image of pattern created using an oxygen plasma etch. Fig. 2(B) shows a higher magnification image from the area indicated in the inset in (B). Above the diamond we see; a $\sim 50 \mathrm{~nm}$ thick amorphous carbon layer formed from the interaction between the diamond and the FIB beam, as well as an additional layer $\sim 10 \mathrm{~nm}$ thick.

Fig. 3 (A) shows an X-ray photoelectron spectrum of this additional layer (from a similarly prepared sample) that indicates that it is composed of the expected species of $\mathrm{C}, \mathrm{O}$ and $\mathrm{Ga}$ (not shown) as well as, unexpectedly, $\mathrm{Fe}$ and $\mathrm{Cr}$. The only potential source of $\mathrm{Fe}$ and $\mathrm{Cr}$ is from ions which have been sputtered from the stainless steel vacuum chamber by the plasma and have selectively re-deposited onto the FIB exposed diamond in a stable manner. The deposition of Fe from vacuum chamber components in a similar plasma environments has been previously observed elsewhere [7].

Fig. 3(B) shows a series of EDX spectra from the surface of a structure patterned using a series of oxygen plasma exposures from 0 to 60 minutes. The X-rays were generated from a $3 \mathrm{kV}$ electron beam which sampled to a depth in the order of the thickness of Fe containing layer. With increased exposure time the Ga-L peak remains approximately constant while peaks from the Fe-L and $\mathrm{O}-\mathrm{K}$ are both seen to increase suggesting that $\mathrm{Fe}$ and $\mathrm{O}$ containing layer is growing.

We propose that the deposition and stable growth of the Fe-containing layer onto the FIB exposed diamond to a thickness significantly greater than the penetration depth of ions from the plasma explains the masking effect observed for the FIBHM. We speculate that this mechanism may be more widely applicable for patterning of other substrates such as silicon. 


\section{References}

[1] W.R. McKenzie et al., Microsc. and Microanal., 15 (2009) 328-329.

[2] A.D. Greentree et al., Mater. Today, 11 (2008) 22-31.

[3] T.M. Babinec et al., Nat Nano, 5 (2010) 195-199.

[4] W.R. McKenzie et al., Diam Relat Mater., (2010) (in submittion).

[5] W.R. McKenzie et al., Microsc. and Microanal, 16 (2010) 196-197.

[6] M.P. Hiscocks et. al., Diam Relat Mater Diamond (2011) (accepted for publication).

[7] T.F. Yen et al., Microelectron Eng, 84 (2007) 1-6.

[8] McKenzie et. al., ACS Nano (2011) (in submission).

[9] Authors would like to acknowledge CRANN at Trinity College Dublin and also the ESTEEM program and Oxford Materials for access to their facilities.
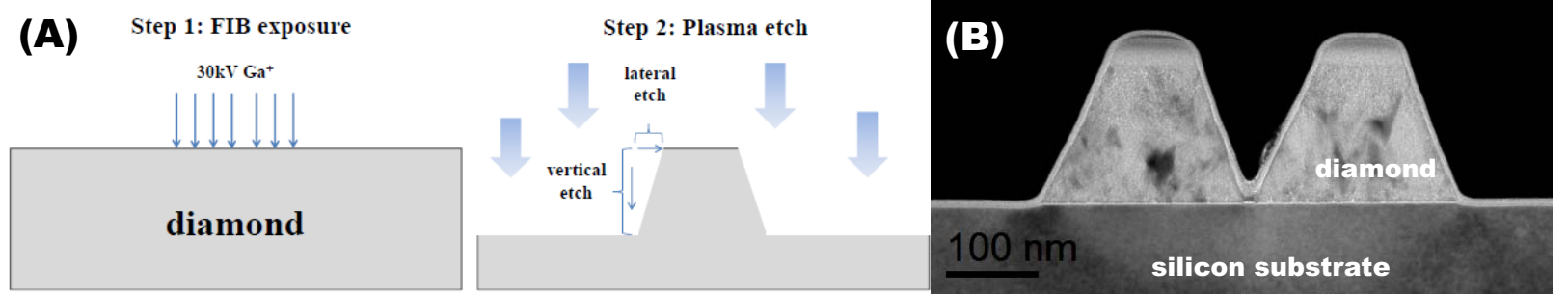

FIG. 1. (A) Schematic of FIBHM process. (B) bright field TEM cross-section showing an example of a structure patterned from a diamond on silicon thin film.
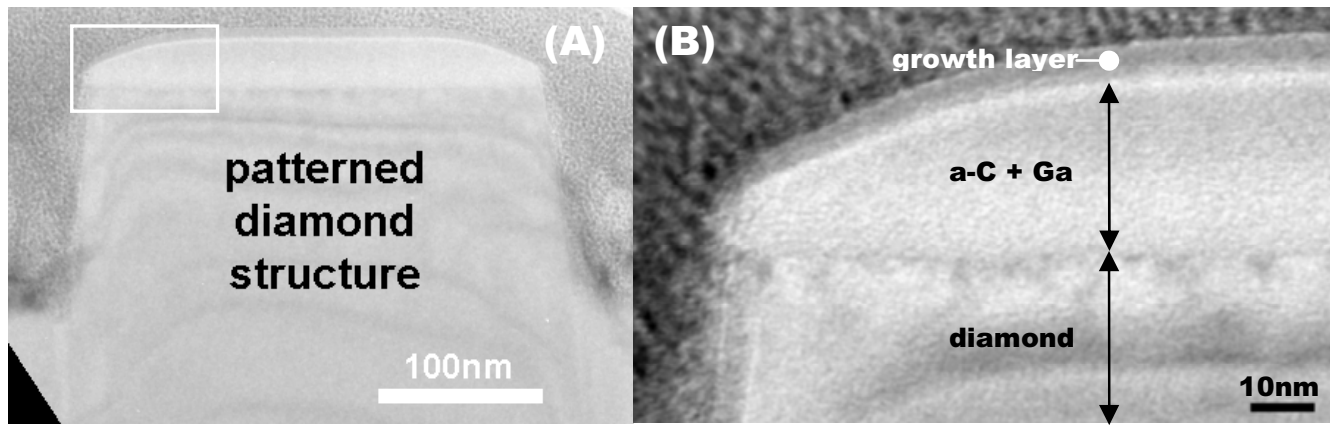

FIG. 2. (A) Bright Field Scanning Transmission Electron Microscope (STEM) image of a cross section of a patterned structure. (B) Bright field STEM image of the inset in (A) highlighting the positions of the diamond, a-C+Ga layer from the Ga-implantation, and additional growth layer.
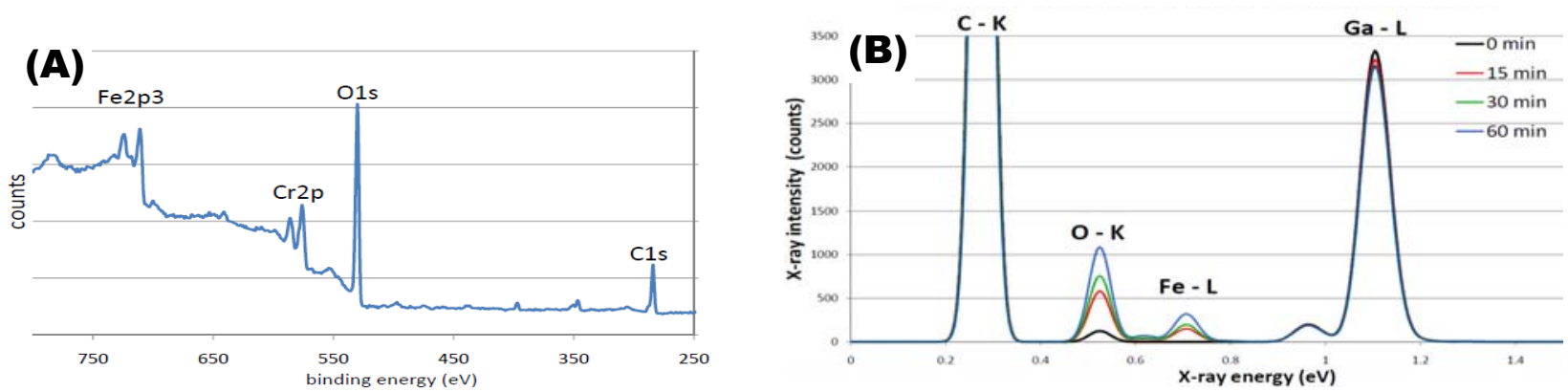

FIG. 3. (A) X-ray photoelectron spectra of taken from the masked surface of a FIBHM patterned structure showing the presence of C, O, Cr and Fe. (B) Energy Dispersive X-Ray spectra taken from the surface of patterned structures which had been exposed to an oxygen plasma etch for $0,15,30$ and 60 minutes [8]. An incident $3 \mathrm{kV}$ electron beam was used such that EDX sampling represented a suitably shallow region of the surface to detect the "growth layer". 